\title{
RIGHLIGHTS
}

ALZHEIMER DISEASE

\section{APP intracellular domain contributes to AD pathogenesis}

Extracellular plaques enriched in amyloid- $\beta(A \beta)$ - a cleavage product of amyloid precursor protein (APP) are widely thought to underlie the pathogenesis of Alzheimer disease (AD). However, recent evidence suggests that $A \beta$ changes are not the whole story. Ghosal et al. report that another APP cleavage product, the APP intracellular domain (AICD), contributes to AD pathology independently of $\mathrm{A} \beta$. "Increased levels of AICD alone, without any changes in $A \beta$, cause agedependent $\mathrm{AD}$-like pathological features in mice," explains principal investigator Sanjay Pimplikar.

Accumulations of $A \beta$ deposits in individuals without dementia indicate that high $A \beta$ loads do not invariably lead to $\mathrm{AD}$. In addition, clinical trials of agents that diminish $A \beta$ deposits have reported disappointing results, indicating that $\mathrm{A} \beta$-independent factors may contribute to AD pathogenesis.

The Pimplikar group used transgenic mice overexpressing AICD that showed abnormal activation of glycogen synthase kinase $3 \beta$ (GSK-3 $\beta$ ), which phosphorylates tau protein. They observed hyperphosphorylation of tau in these transgenic mice when compared with wild-type mice, an effect that increased with age. The investigators also noted more aggregation of tau in

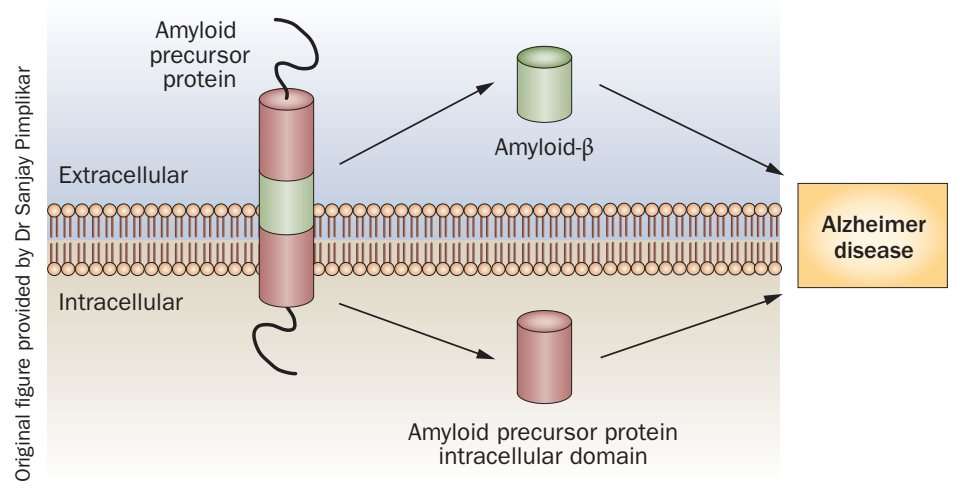

$\geq 8$ month-old transgenic mice than in controls. Enhanced aggregation was not seen in 4 month-old transgenic mice, indicating a lag between AICD-dependent tau phosphorylation and formation of hyperphosphorylated tau aggregates.

Most mouse models of AD lack neurodegeneration, but AICD transgenic mice aged $>18$ months showed a significant loss of neurons in the hippocampal CA3 region. This CA3 neurodegeneration could also be induced in 4 month-old transgenic mice, but not in controls, by administering a subconvulsive dose of kainic acid, which promotes excitotoxic shock. Furthermore, the investigators observed age-dependent memory losses in transgenic mice that could be reversed by GSK-3 $\beta$ inhibition (and hence reduced tau phosphorylation) with lithium.

The investigators also found considerably higher levels of AICD and phosphorylated tau in 13 brain samples from patients with $\mathrm{AD}$ compared with those from 12 healthy individuals, an observation that highlights the clinical relevance of this work. "We are currently characterizing the AICD effects at the molecular level to identify novel drug targets that do not lie on the amyloid pathway," concludes Pimplikar.

\section{Rowan Higgs}

Original article Ghosal, K. et al. Alzheimer's disease-lik pathological features in transgenic mice expressing the APP intracellular domain. Proc. Natl Acad. Sci. USA 106, 18367-18372 (2009) 